\title{
An Age-structured Model of Bird Migration
}

\author{
S.A. Gourley ${ }^{1} *$, Rongsong Liu $^{2}$ \\ ${ }^{1}$ Department of Mathematics, University of Surrey, Guildford, Surrey, GU2 7XH, UK \\ ${ }^{2}$ Department of Mathematics and Department of Zoology and Physiology \\ University of Wyoming, Laramie, WY, USA, 82071
}

\begin{abstract}
An approach to modelling bird migration is proposed, in which there is a region where birds do not move but spend time breeding. Birds leave this breeding region and enter a migration flyway which is effectively a one-way corridor starting and ending at the breeding location. Mathematically, the flyway is a curve parametrised by arc-length. Flight speed depends on position along the flyway, to take account of factors such as wind and the pausing of birds at various locations for wintering or stopovers. Per-capita mortality along the flyway is both position and age-dependent, allowing for increased risks at stopover locations due to predation, and increased risks to immature birds. A reaction-advection age-structured equation models population dynamics along the flyway and, using a Laplace transform technique, the model is reduced to a scalar delay differential equation for the number of adult birds at the breeding location. Extinction and persistence criteria are obtained for the bird population and the results of computer simulations are presented.
\end{abstract}

Keywords and phrases: bird migration, age-structure, advection, delay, stability

Mathematics Subject Classification: 34K20, 34K25, 34K60, 92D40

\section{Introduction and preliminaries}

In this paper we present an approach to modelling a population of migratory birds. Such birds migrate long distances along flyways, usually northwards in the spring to breeding grounds in temperate or Arctic regions, and then southwards in the autumn to wintering grounds in warmer regions. For example, the rufous hummingbird breeds in locations including southern Alaska and, in autumn, migrates south along a flyway following the Rocky Mountains to Mexico for winter. The European pied flycatcher breeds in many parts of Europe and migrates to wintering grounds in west Africa. The bar-headed goose breeds in Central Asia and migrates over the Himalayas to wintering grounds in South Asia. The northern pintail breeds at locations in many northerly latitudes including northern Europe, Canada and parts of the USA. Its wintering grounds are in central America and tropical parts of Africa and Asia.

In this paper the location where the birds breed in the summer is considered as a two dimensional finite region and will be referred to as the summer breeding patch, with a subscript $b$ (meaning breeding) used for variables and parameters that relate to that patch. We do not consider the motion of birds within the breeding patch but we consider age-dependence. We introduce the variable $S_{b}(t, a)$, which does not involve space, to denote the density of birds of age $a$ at time $t$ in the summer breeding patch

\footnotetext{
*Corresponding author. E-mail: s.gourley@surrey.ac.uk
} 
$b$. In the autumn, birds leave the breeding patch to begin migration. Of course, we do consider motion of birds along the migration flyway, via the use of reaction-advection theory since birds effectively fly in a particular direction along a one-way corridor. We derive a partial differential equation for $S_{b}(t, a)$, equation (2.6). The model is reduced to a scalar delay differential equation (equation (2.15)) for the number $S_{b}^{\text {ad }}(t)$ of adult birds at the summer breeding patch. The reduction is not straightforward since it requires the solution of (2.6) which, though linear, is not easy to solve. Our approach is via Laplace transforms.

As shown in Fig. 1, birds leave the breeding patch in the autumn to begin their migration to warmer climates for winter, after which they return to the summer breeding location by another route. There will be stopover locations along the route where birds rest and eat. Unlike in our previous works $[1,5]$, in this paper the location where birds spend winter, and the stopover locations, are not considered as separate patches. Each such location is simply interpreted as a particular interval of the flyway, or even a single point on it. The whole flyway is modelled as a one-dimensional curve starting and ending at the summer breeding location. In reality a bird will pause at the wintering or at a stopover location, but for purposes of the modelling we suppose that it instead passes through, with a greatly reduced velocity, an interval on the flyway containing the place where in reality it pauses. Increased per-capita mortality in these intervals can model the increased risk of predation, from ground-based predators such as snakes, that birds may face when they pause anywhere. The summer breeding location $b$ is not considered a part of the flyway.

A spatially extended model, in the form of a reaction-advection equation (see (2.2) below), is used to model the migration and all bird population dynamics other than at the summer breeding patch $b$. The use of a reaction-advection (rather than a reaction-diffusion) equation to describe the migration is appropriate since the birds effectively fly along a one-way corridor that we call the migration flyway. In this paper the migration flyway is modelled mathematically as a curve parametrised by arc length $x$, in other words, $x$ is defined as the distance along the curve from the location at which the birds begin their autumn migration. The loop starts at $x=0$, the starting point of the autumn migration, and ends at $x=L$ where birds arrive back at the summer breeding location at the end of the spring migration. The wintering and stopover locations are just particular points of the loop, or intervals along it. The idea of a migration loop, with a single spatial variable denoting arc length, creates a model with just one spatial dimension whilst also allowing outward and return migration routes to be different. In fact in North America the loop is often traversed in the clockwise sense.

Since the flyway is mathematically a one dimensional curve, we define the positive, scalar-valued, differentiable function $U(x)$ to be the birds' flight speed at location $x$ along the flyway. The need for $U$ to depend on $x$ is obvious for two reasons. Firstly, flight speed depends on location-dependent factors such as the wind. Secondly, birds are envisioned to pass through, with a greatly reduced velocity, intervals of the flyway that correspond to wintering and stopover locations where in reality they would pause. At points on the flyway, $\mu_{\mathrm{fly}}(a, x)$ is the birds' per-capita mortality rate which depends on age $a$ and on flyway location $x$, since in some parts of the migration flyway they are vulnerable to attack by predatory birds or, in the case of wintering and stopover locations, land-based predators such as snakes.

\section{Model derivation}

For points on the flyway shown in Fig. 1, let $s(t, a, x)$ denote the density of birds at time $t$, age $a$, and location $x$ along the flyway, with $x \in[0, L]$. Standard theory yields that $s(t, a, x)$ satisfies an equation of the form

$$
\frac{\partial s(t, a, x)}{\partial t}+\frac{\partial s(t, a, x)}{\partial a}=-\frac{\partial J(t, a, x)}{\partial x}-\mu_{\mathrm{fly}}(a, x) s(t, a, x),
$$

where $J(t, a, x)$ is the flux of birds of age $a$ (the rate at time $t$ at which age $a$ birds pass location $x$ ). On the assumption that each bird flies in the positive $x$ direction at a prescribed speed $U(x)$, the flux at $x$ 


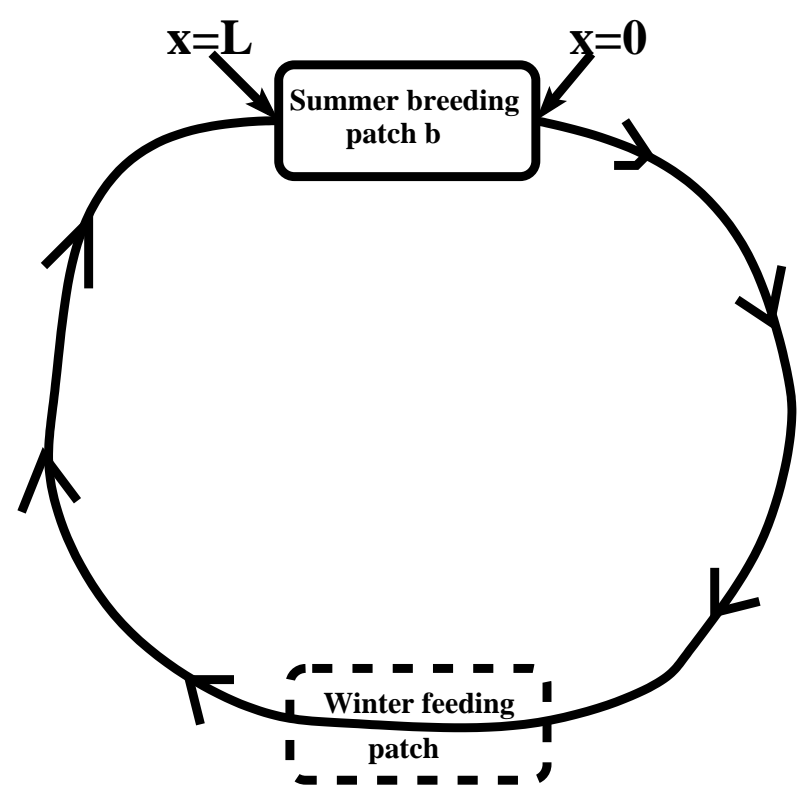

Figure 1. Migration route. Birds leave the summer breeding patch $b$ at $x=0$, the starting point of the autumn migration. They finish the spring migration by returning to the breeding patch at $x=L$. The migration route $x \in[0, L]$ includes the autumn and spring migrations, and also the wintering and any stopover locations which are interpreted as particular intervals or points along the curve.

TABLE 1. Parameters and parameter functions.

\begin{tabular}{|c|c|c|}
\hline para. & definition, unit & value (likely range) \\
\hline$D$ & migration rate of birds, day ${ }^{-1}$ & variable $\in(0,0.15)$ \\
\hline$\mu_{b}^{\mathrm{ad}}$ & death rate of adult birds in patch $b$, day $^{-1}$ & $0.0005[1 /(10 \times 365), 1 /(5 \times 365)]$ \\
\hline$\mu_{b}^{\mathrm{juv}}$ & death rate of juvenile birds in patch $b$, day $^{-1}$ & $3 \times \mu_{b}^{\mathrm{ad}}$ \\
\hline$\mu_{\mathrm{fly}}^{\mathrm{ad}}$ & per-capita flyway death rate of adult birds, day ${ }^{-1}$ & 0.002 \\
\hline$\mu_{\text {fly }}^{\text {juv }}$ & per-capita flyway death rate of juvenile birds, day ${ }^{-1}$ & $2 \times \mu_{\mathrm{fly}}^{\mathrm{ad}}$ \\
\hline$\tau$ & duration of juvenile stage of birds, days & 365 \\
\hline$L$ & $\begin{array}{l}\text { total length of autumn migration, winter patch } \\
\text { and spring migration, miles }\end{array}$ & 1600 \\
\hline$r$ & $\begin{array}{l}\text { maximum possible per-capita female egg } \\
\text { production rate }\end{array}$ & 3 \\
\hline $1 / k$ & $\begin{array}{l}\text { population size at which the bird population as a } \\
\text { whole achieves maximum reproductive success }\end{array}$ & 10000 \\
\hline$B(\cdot)$ & birth rate function & see $(2.10)$ \\
\hline$U(x)$ & flight speed at location $x \in[0, L]$, miles per day & see $(4.1)$ \\
\hline$U_{0}$ & flight speed when not pausing, miles per day & 100 \\
\hline
\end{tabular}

is given by $J(t, a, x)=U(x) s(t, a, x)$. Therefore, (2.1) becomes

$$
\frac{\partial s(t, a, x)}{\partial t}+\frac{\partial s(t, a, x)}{\partial a}=-U(x) \frac{\partial s(t, a, x)}{\partial x}-\left(\mu_{\mathrm{fly}}(a, x)+U^{\prime}(x)\right) s(t, a, x), \quad x \in(0, L) .
$$

Equation (2.2) is a reaction advection equation. At time $t$ the flux at $x=0$ of age $a$ birds (i.e. flux of age $a$ birds leaving patch $b$ at the start of migration) is $U(0) s(t, a, 0)$. These birds will return some time later, if they survive the migration. We calculate the flux $U(L) s(t, a, L)$ of birds of age $a$ returning to 
patch $b$ at time $t$ after their migration, in terms of the flux of those same birds as they left patch $b$ at the earlier time $t-G(L)$, with $G(\cdot)$ defined in (2.3), and correspondingly younger age $a-G(L)$. This expression turns out to be $(2.5)$ below, as we now show.

Let

$$
s^{\xi}(a, x)=s(a+\xi, a, x)
$$

Then, by $(2.2), s^{\xi}(a, x)$ satisfies

$$
\frac{\partial s^{\xi}(a, x)}{\partial a}=-U(x) \frac{\partial s^{\xi}(a, x)}{\partial x}-\left(\mu_{\mathrm{fly}}(a, x)+U^{\prime}(x)\right) s^{\xi}(a, x) .
$$

Denoting

$$
y(a, x)=s^{\xi}(a, x) \quad \text { and } \quad G(x)=\int_{0}^{x} \frac{d \tilde{x}}{U(\tilde{x})}
$$

we have

$$
G^{\prime}(x) \frac{\partial y(a, x)}{\partial a}+\frac{\partial y(a, x)}{\partial x}=-\frac{\left(\mu_{\mathrm{fl}}(a, x)+U^{\prime}(x)\right)}{U(x)} y(a, x) .
$$

In (2.3) we assume that $U$ is never zero, even though birds may pause at stopovers such as the winter feeding patch. We deal with this issue by taking $U$ to be very small in regions of the flyway where birds in reality would pause. An example of an appropriate non-zero choice for $U(x)$ is given in expression (4.1). Let

$$
y^{\eta}(x)=y(\eta+G(x), x) .
$$

Then $y^{\eta}(x)$ satisfies

$$
\frac{d y^{\eta}(x)}{d x}=-\frac{\left(\mu_{\mathrm{fly}}(\eta+G(x), x)+U^{\prime}(x)\right)}{U(x)} y^{\eta}(x) .
$$

Integrating from 0 to $x$, and using (2.4), we obtain

$$
y(\eta+G(x), x)=y(\eta, 0) \exp \left\{-\int_{0}^{x} \frac{\mu_{\mathrm{fly}}(\eta+G(\bar{x}), \bar{x})+U^{\prime}(\bar{x})}{U(\bar{x})} d \bar{x}\right\} .
$$

Setting $\eta=a-G(x)$, we have

$$
s^{\xi}(a, x)=y(a, x)=y(a-G(x), 0) \exp \left\{-\int_{0}^{x} \frac{\mu_{\mathrm{fly}}(a-G(x)+G(\bar{x}), \bar{x})+U^{\prime}(\bar{x})}{U(\bar{x})} d \bar{x}\right\} .
$$

Since $s^{\xi}(a, x)=s(a+\xi, a, x)$,

$$
\begin{aligned}
& s(a+\xi, a, x)= \\
& s^{\xi}(a-G(x), 0) \exp \left\{-\int_{0}^{x} \frac{\mu_{\mathrm{fly}}(a-G(x)+G(\bar{x}), \bar{x})+U^{\prime}(\bar{x})}{U(\bar{x})} d \bar{x}\right\} \\
& =s(a-G(x)+\xi, a-G(x), 0) \exp \left\{-\int_{0}^{x} \frac{\mu_{\mathrm{fly}}(a-G(x)+G(\bar{x}), \bar{x})+U^{\prime}(\bar{x})}{U(\bar{x})} d \bar{x}\right\} .
\end{aligned}
$$

Setting $x=L$ and $\xi=t-a$, we have

$$
s(t, a, L)=s(t-G(L), a-G(L), 0) \exp \left\{-\int_{0}^{L} \frac{\mu_{\mathrm{fly}}(a-G(L)+G(\bar{x}), \bar{x})+U^{\prime}(\bar{x})}{U(\bar{x})} d \bar{x}\right\} .
$$

Recall that $S_{b}(t, a)$ is the age density of birds in the summer breeding patch $b$. Recall the general expression for the flux, $J(t, a, x)=U(x) s(t, a, x)$. Assume that the flux out of patch $b$ is $D S_{b}(t, a)$, so 
that $D$ stands for the per-capita departure rate. The rate at that time at which older birds re-enter the summer breeding patch $b$ at the end of their migration cycle is $U(L) s(t, a, L)$, given by

$$
\begin{aligned}
& U(L) s(t, a, L) \\
& =U(L) s(t-G(L), a-G(L), 0) \exp \left\{-\int_{0}^{L} \frac{\mu_{\mathrm{fly}}(a-G(L)+G(\bar{x}), \bar{x})+U^{\prime}(\bar{x})}{U(\bar{x})} d \bar{x}\right\} .
\end{aligned}
$$

But $s(t-G(L), a-G(L), 0)=J(t-G(L), a-G(L), 0) / U(0)$ and $J(t-G(L), a-G(L), 0)$ is the flux at time $t-G(L)$ of age $a-G(L)$ individuals at location $x=0$ (i.e. the flux out of patch $b$ ), and is therefore given by $D S_{b}(t-G(L), a-G(L))$. Therefore

$$
\begin{aligned}
& U(L) s(t, a, L) \\
& =\frac{U(L)}{U(0)} D S_{b}(t-G(L), a-G(L)) \exp \left\{-\int_{0}^{L} \frac{\mu_{\mathrm{fly}}(a-G(L)+G(\bar{x}), \bar{x})+U^{\prime}(\bar{x})}{U(\bar{x})} d \bar{x}\right\} \\
& =D S_{b}(t-G(L), a-G(L)) \exp \left\{-\int_{0}^{L} \frac{\mu_{\mathrm{fly}}(a-G(L)+G(\bar{x}), \bar{x})}{U(\bar{x})} d \bar{x}\right\} .
\end{aligned}
$$

Therefore, $S_{b}(t, a)$ satisfies, for $a>G(L)$ and $t>G(L)$,

$$
\begin{aligned}
& \frac{\partial S_{b}(t, a)}{\partial t}+\frac{\partial S_{b}(t, a)}{\partial a}=-\mu_{b}(a) S_{b}(t, a)-D S_{b}(t, a) \\
& +D S_{b}(t-G(L), a-G(L)) \exp \left\{-\int_{0}^{L} \frac{\mu_{\mathrm{fly}}(a-G(L)+G(\bar{x}), \bar{x})}{U(\bar{x})} d \bar{x}\right\},
\end{aligned}
$$

where $\mu_{b}(a)$ is the mortality rate experienced by the birds when they are at the summer breeding patch $b$, which we take to be of the form

$$
\mu_{b}(a)=\left\{\begin{array}{l}
\mu_{b}^{\mathrm{ad}}, a>\tau, \\
\mu_{b}^{\mathrm{juv}}, a<\tau,
\end{array}\right.
$$

where the superscripts ad and juv stand for adult and juvenile, and $\tau$ is the age at which a bird reaches maturation. For the per-capita flyway mortality rate $\mu_{\mathrm{fly}}(a, x)$, we take

$$
\mu_{\mathrm{fly}}(a, x)=\left\{\begin{array}{l}
\mu_{\mathrm{fly}}^{\mathrm{ad}}(x), a>\tau, \\
\mu_{\mathrm{fly}}^{\mathrm{juv}}(x), a<\tau .
\end{array}\right.
$$

The last two terms in the right hand side of (2.6) are the flux terms, with $D S_{b}(t, a)$ being the rate that birds depart from the breeding patch for the autumn migration, and the last term being the rate at which they return after the spring migration. This term involves the departure rate $D S_{b}(t-G(L), a-G(L))$ at the earlier time $t-G(L)$ where $G(L)=\int_{0}^{L} d \tilde{x} / U(\tilde{x})$ is the total journey time, since the integrand is the time to travel an incremental distance $d \tilde{x}$. The exponential in the last term of (2.6) is the probability that a bird survives the whole migration including wintering and stopovers. Survival probabilities often assume the form $\exp (-\mu \tau)$ where $\mu$ is the mortality rate through some stage and $\tau$ is the length of that stage. The exponential in (2.6) is essentially of that form since $d \bar{x} / U(\bar{x})$ is a time variable, representing the time to travel an increment of the flyway of length $d \bar{x}$. The only remaining issue that needs an interpretation is why the first argument of $\mu_{\mathrm{fly}}$ in the integrand is $a-G(L)+G(\bar{x})$. But a bird that is of age $a$ when it returns at time $t$ was of age $a-G(L)$ when it departed. The time that bird took to travel from $x=0$ to $x=\bar{x}$ is $G(\bar{x})$. That bird was therefore of age $a-G(L)+G(\bar{x})$ when it passed the point $\bar{x}$ on the route. The first argument of $\mu_{\mathrm{fly}}$ in the integrand simply keeps track of the age of a bird at each stage $\bar{x} \in[0, L]$ of its journey. 
The total number $S_{b}^{\text {ad }}(t)$ of adult birds at the summer breeding patch $b$ is given by integrating $S_{b}(t, a)$ over all ages $a$ that constitute adulthood, so that

$$
S_{b}^{\mathrm{ad}}(t)=\int_{\tau}^{\infty} S_{b}(t, a) d a
$$

and we assume that the birth rate $S_{b}(t, 0)$ satisfies

$$
S_{b}(t, 0)=B\left(S_{b}^{\mathrm{ad}}(t)\right),
$$

where $B(\cdot)$ is a prescribed function of the total number of adult birds $S_{b}^{\mathrm{ad}}(t)$ at the breeding patch $b$. Differentiating (2.9) with respect to $t$ and using (2.6) and (2.7) gives

$$
\begin{aligned}
& \frac{d S_{b}^{\mathrm{ad}}(t)}{d t}=S_{b}(t, \tau)-\left(\mu_{b}^{\mathrm{ad}}+D\right) S_{b}^{\mathrm{ad}}(t) \\
& +D \int_{\tau}^{\infty} S_{b}(t-G(L), a-G(L)) \exp \left\{-\int_{0}^{L} \frac{\mu_{\mathrm{fly}}(a-G(L)+G(\bar{x}), \bar{x})}{U(\bar{x})} d \bar{x}\right\} d a \\
& =S_{b}(t, \tau)-\left(\mu_{b}^{\mathrm{ad}}+D\right) S_{b}^{\mathrm{ad}}(t) \\
& +D \int_{\tau}^{\tau+G(L)} S_{b}(t-G(L), a-G(L)) \exp \left\{-\int_{0}^{L} \frac{\mu_{\mathrm{fly}}(a-G(L)+G(\bar{x}), \bar{x})}{U(\bar{x})} d \bar{x}\right\} d a \\
& +D \int_{\tau}^{\infty} S_{b}(t-G(L), \bar{a}) \exp \left\{-\int_{0}^{L} \frac{\mu_{\mathrm{fly}}(\bar{a}+G(\bar{x}), \bar{x})}{U(\bar{x})} d \bar{x}\right\} d \bar{a} .
\end{aligned}
$$

In the last integral the flyway mortality $\mu_{\mathrm{fly}}(\bar{a}+G(\bar{x}), \bar{x})$ only involves adult ages and therefore, using $(2.8)$, that last integral can be written as

$$
D \exp \left(-\int_{0}^{L} \frac{\mu_{\mathrm{fl}}^{\mathrm{ad}}(\bar{x})}{U(\bar{x})} d \bar{x}\right) S_{b}^{\mathrm{ad}}(t-G(L)),
$$

and therefore

$$
\begin{aligned}
& \frac{d S_{b}^{\mathrm{ad}}(t)}{d t}=S_{b}(t, \tau)-\left(\mu_{b}^{\mathrm{ad}}+D\right) S_{b}^{\mathrm{ad}}(t) \\
& +D \int_{\tau}^{\tau+G(L)} S_{b}(t-G(L), a-G(L)) \exp \left\{-\int_{0}^{L} \frac{\mu_{\mathrm{fly}}(a-G(L)+G(\bar{x}), \bar{x})}{U(\bar{x})} d \bar{x}\right\} d a \\
& +D \exp \left(-\int_{0}^{L} \frac{\mu_{\mathrm{fly}}^{\mathrm{ad}}(\bar{x})}{U(\bar{x})} d \bar{x}\right) S_{b}^{\mathrm{ad}}(t-G(L)) .
\end{aligned}
$$

Equation (2.11) describes the rate of change of the number of adult birds $S_{b}^{\text {ad }}(t)$ in the breeding patch $b$ at time $t$. The term $S_{b}(t, \tau)$ is the maturation rate for birds that mature in the breeding patch (as opposed to somewhere along the flyway) and $\mu_{b}^{\mathrm{ad}} S_{b}^{\mathrm{ad}}(t)$ is the death rate on the breeding patch. The term $-D S_{b}^{\text {ad }}(t)$ is the rate at which adult birds leave the breeding patch to begin a migration (juvenile birds may migrate as well, but this is accounted for more indirectly since (2.11) describes the change rate for adult birds only). The last two terms together represent the rate at which adult birds arrive at the breeding patch at the end of a migration. Note that both terms involve the departure rate at the earlier time $t-G(L)$ when those birds began the migration. In both terms the exponentials represent the probability of surviving the migration. The term on the second line of (2.11) represents the arrival rate of birds that are adults when they return at time $t$ but were juveniles, of various ages between $\tau-G(L)$ and $\tau$, when they began the migration at time $t-G(L)$. The last term in (2.11) is the rate of return for 
birds that were already adults when they began the migration at time $t-G(L)$. Since $(2.11)$ describes the change rate for adult birds only, there is no term for arriving birds that remained juvenile throughout a migration cycle.

We derive a delay differential equation for the variable $S_{b}^{\text {ad }}$. This can be achieved by solving (2.6) for $S_{b}(t, a)$, as follows. Defining $S_{b}^{\zeta}(a)=S_{b}(\zeta+a, a)$, we find from $(2.6)$ that $S_{b}^{\zeta}(a)$ satisfies

$$
\begin{aligned}
\frac{d S_{b}^{\zeta}(a)}{d a} & =-\mu_{b}(a) S_{b}^{\zeta}(a)-D S_{b}^{\zeta}(a) \\
& +D S_{b}^{\zeta}(a-G(L)) \exp \left\{-\int_{0}^{L} \frac{\mu_{\mathrm{fly}}(a-G(L)+G(\bar{x}), \bar{x})}{U(\bar{x})} d \bar{x}\right\} .
\end{aligned}
$$

We solve (2.12) for $S_{b}^{\zeta}(a)$, for ages $a \leq \tau$, by a Laplace transform technique. Laplace transforms involve integration over $a \in[0, \infty)$ so in fact we apply the Laplace transform to a certain modified equation, namely, equation $(2.12)$ with $\mu_{b}(a)$ replaced by $\mu_{b}^{\text {juv }}$, and $\mu_{\mathrm{fly}}(a-G(L)+G(\bar{x}), \bar{x})$ by $\mu_{\mathrm{fly}}^{\mathrm{juv}}(\bar{x})$. This modified equation is not, of course, correct for all ages $a$. However, the solutions of (2.12) and the modified problem coincide for $a \leq \tau$ which is all we need. With this understanding, applying the Laplace transform gives

$$
\begin{aligned}
p \hat{S}_{b}^{\zeta}(p)-S_{b}^{\zeta}(0) & =-\left(\mu_{b}^{\mathrm{juv}}+D\right) \hat{S}_{b}^{\zeta}(p) \\
& +D \exp \left(-\int_{0}^{L} \frac{\mu_{\mathrm{fly}}^{\mathrm{juv}}(\bar{x})}{U(\bar{x})} d \bar{x}\right) \int_{0}^{\infty} S_{b}^{\zeta}(a-G(L)) e^{-p a} d a
\end{aligned}
$$

where the hat denotes Laplace transform and $p$ is the transform variable. The integral can be put in the form

$$
e^{-p G(L)}\left[\int_{-G(L)}^{0} S_{b}^{\zeta}(a) e^{-p a} d a+\hat{S}_{b}^{\zeta}(p)\right]
$$

and therefore, recalling that $S_{b}^{\zeta}(a)=S_{b}(\zeta+a, a)$,

$$
\hat{S}_{b}^{\zeta}(p)=\frac{S_{b}(\zeta, 0)+D e^{-p G(L)} \exp \left(-\int_{0}^{L} \frac{\mu_{\mathrm{fly}}^{\mathrm{juv}}(\bar{x})}{U(\bar{x})} d \bar{x}\right) \int_{-G(L)}^{0} S_{b}(\zeta+a, a) e^{-p a} d a}{p+\mu_{b}^{\mathrm{juv}}+D-D e^{-p G(L)} \exp \left(-\int_{0}^{L} \frac{\mu_{\mathrm{fly}}^{\mathrm{juv}}(\bar{x})}{U(\bar{x})} d \bar{x}\right)} .
$$

We take $S_{b}(t, a)=0$ for $a<0$, so only the $S_{b}(\zeta, 0)$ term in the numerator survives. Using the birth law (2.10), where the function $B(\cdot)$ is introduced, and applying the standard inversion formula for the Laplace transform involving a contour integral using a standard Bromwich contour $\Gamma$,

$$
S_{b}^{\zeta}(a)=\frac{1}{2 \pi i} \int_{\Gamma} \frac{B\left(S_{b}^{\mathrm{ad}}(\zeta)\right) e^{p a} d p}{p+\mu_{b}^{\mathrm{juv}}+D-D e^{-p G(L)} \exp \left(-\int_{0}^{L} \frac{\mu_{\mathrm{fly}}^{\mathrm{juv}}(\bar{x})}{U(\bar{x})} d \bar{x}\right)},
$$

and therefore $S_{b}^{\zeta}(a)$ can be expressed as the sum of the residues of the poles of $\hat{S}_{b}^{\zeta}(p)$, which are the zeroes of its denominator. Precisely one of these zeroes is real; we denote it by $p^{*}$. It is easy to show that $p^{*}<0$. All other zeroes are complex with real part less than $p^{*}$. Retaining only the pole at $p=p^{*}$, computing the residue of the integrand at that pole and applying Cauchy's residue theorem, we obtain

$$
S_{b}^{\zeta}(a) \approx \frac{B\left(S_{b}^{\mathrm{ad}}(\zeta)\right) e^{p^{*} a}}{1+D G(L) e^{-p^{*} G(L)} \exp \left(-\int_{0}^{L} \frac{\mu_{\mathrm{fly}}^{\mathrm{juv}}(\bar{x})}{U(\bar{x})} d \bar{x}\right)}
$$


on the assumption that the residues at the other poles are small compared to the residue at $p=p^{*}$, which is reasonable since those other poles have smaller real parts. Setting $\zeta=t-a$, and using that $p^{*}$ satisfies

$$
p^{*}+\mu_{b}^{\mathrm{juv}}+D=D e^{-p^{*} G(L)} \exp \left(-\int_{0}^{L} \frac{\mu_{\mathrm{fly}}^{\mathrm{juv}}(\bar{x})}{U(\bar{x})} d \bar{x}\right)
$$

we obtain

$$
S_{b}(t, a) \approx \frac{B\left(S_{b}^{\mathrm{ad}}(t-a)\right) e^{p^{*} a}}{1+G(L)\left(p^{*}+\mu_{b}^{\mathrm{juv}}+D\right)} .
$$

This is for $t>a$ and ages $a \leq \tau$. Finally, the function $S_{b}$ is eliminated from equation (2.11) using the approximation (2.14) to obtain that $S_{b}^{\text {ad }}(t)$ evolves approximately according to the delay differential equation

$$
\begin{aligned}
& \frac{d S_{b}^{\mathrm{ad}}(t)}{d t}=\frac{B\left(S_{b}^{\mathrm{ad}}(t-\tau)\right) e^{p^{*} \tau}}{1+G(L)\left(p^{*}+\mu_{b}^{\mathrm{juv}}+D\right)}-\left(\mu_{b}^{\mathrm{ad}}+D\right) S_{b}^{\mathrm{ad}}(t) \\
& +D \int_{\tau}^{\tau+G(L)} \frac{B\left(S_{b}^{\mathrm{ad}}(t-a)\right) e^{p^{*}(a-G(L))}}{1+G(L)\left(p^{*}+\mu_{b}^{\mathrm{juv}}+D\right)} \exp \left\{-\int_{0}^{L} \frac{\mu_{\mathrm{fly}}(a-G(L)+G(\bar{x}), \bar{x})}{U(\bar{x})} d \bar{x}\right\} d a \\
& +D \exp \left(-\int_{0}^{L} \frac{\mu_{\mathrm{fly}}^{\mathrm{ad}}(\bar{x})}{U(\bar{x})} d \bar{x}\right) S_{b}^{\mathrm{ad}}(t-G(L))
\end{aligned}
$$

where $p^{*}$ is the unique real root of (2.13). Recall that $p^{*}<0$.

\subsection{Remarks on the maturation rate}

The term $S_{b}(t, \tau)$ in $(2.11)$ is the maturation rate for birds that mature in the breeding patch (some birds might mature while in migration). We have computed an approximation for this term, the first term in the right hand side of (2.15). Here, we make a few remarks about that approximation with a view to elucidating the circumstances under which it is likely to be most accurate. From a mathematical point of view, we might ask under what circumstances the residues of the poles other than at $p=p^{*}$ will be negligible. A glance at (2.13) suggests that this will be the case if $G(L)$, which is the total duration of a migration, is small. This is because decreasing $G(L)$ in $(2.13)$ will force the non-real poles into the far left of the complex plane. We will measure smallness of $G(L)$ relative to the maturation time $\tau$, thereby introducing a small parameter $\epsilon$ further down.

Before doing so, however, we derive another expression for $S_{b}(t, \tau)$ based on purely probabilistic reasoning. Either from probabilistic reasoning or Laplace transform reasoning, we have that $S_{b}(t, \tau)$ equals $B\left(S_{b}^{\text {ad }}(t-\tau)\right)$ multiplied by the birth to adult survival probability for a bird, given that it matures in the breeding patch $b$. Any bird is, of course, born in that patch. Suppose a bird completes $n$ migration cycles whilst a juvenile bird. We assert that the birth to adult survival probability is

$$
e^{-\mu_{b}^{\mathrm{juv}}(\tau-n G(L))} \exp \left(-n \int_{0}^{L} \frac{\mu_{\mathrm{fly}}^{\mathrm{juv}}(\bar{x})}{U(\bar{x})} d \bar{x}\right) .
$$

If a bird had spent all its time as a juvenile in the breeding patch this probability would be $e^{-\mu_{b}^{\text {juv }} \tau}$. However, total time spent in migration is $n G(L)$ so time spent in the breeding patch is $\tau-n G(L)$, during which time the birds experience a per-capita mortality of $\mu_{b}^{\text {juv }}$ giving rise to the factor $e^{-\mu_{b}^{\text {juv }}(\tau-n G(L))}$. The second exponential factor in (2.16), in the case $n=1$, is the probability of surviving a single migration. With $n$ present, that factor is the probability of surviving all $n$ migrations that happen while the bird is a juvenile. The mean time spent in the breeding patch between arriving there and departing for the next migration is $1 / D$. Therefore, $n G(L)+n / D=\tau$ and

$$
n=\frac{\tau}{G(L)+D^{-1}} .
$$


The survival probability in $(2.16)$ is therefore

$$
\exp \left\{-\left(\frac{\tau}{G(L)+D^{-1}}\right)\left[\frac{\mu_{b}^{\mathrm{juv}}}{D}+\int_{0}^{L} \frac{\mu_{\mathrm{fly}}^{\mathrm{juv}}(\bar{x})}{U(\bar{x})} d \bar{x}\right]\right\}
$$

and the corresponding survival probability from $(2.15)$ is

$$
\frac{e^{p^{*} \tau}}{1+G(L)\left(p^{*}+\mu_{b}^{\mathrm{juv}}+D\right)}
$$

with $p^{*}$ from (2.13).

We now compare (2.18) with (2.19). As explained earlier we introduce a small parameter $\epsilon$ defined by $\epsilon=G(L) / \tau$. So $L$ now depends on $\epsilon$ and we write $L(\epsilon)$, where $G(L(\epsilon))=\tau \epsilon$. Recall that $G$ is defined in (2.3). Also $p^{*}$ in (2.19) depends on $\epsilon$ so we write $p^{*}(\epsilon)$ which satisfies

$$
p^{*}(\epsilon)+\mu_{b}^{\mathrm{juv}}+D=D e^{-p^{*}(\epsilon) \tau \epsilon} \exp \left(-\int_{0}^{L(\epsilon)} \frac{\mu_{\mathrm{fly}}^{\mathrm{juv}}(\bar{x})}{U(\bar{x})} d \bar{x}\right) .
$$

Note that $G(L(0))=0$ and so $L(0)=0$. Therefore, $p^{*}(0)=-\mu_{b}^{\text {juv }}$. In terms of $\epsilon$, expressions $(2.18)$ and (2.19) become

$$
P(\epsilon)=\exp \left\{-\left(\frac{\tau}{\tau \epsilon+D^{-1}}\right)\left[\frac{\mu_{b}^{\mathrm{juv}}}{D}+\int_{0}^{L(\epsilon)} \frac{\mu_{\mathrm{fly}}^{\mathrm{juv}}(\bar{x})}{U(\bar{x})} d \bar{x}\right]\right\}
$$

and

$$
Q(\epsilon)=\frac{e^{p^{*}(\epsilon) \tau}}{1+\tau \epsilon\left(p^{*}(\epsilon)+\mu_{b}^{\text {juv }}+D\right)} .
$$

Note that $P(0)=Q(0)=e^{-\mu_{b}^{\mathrm{juv}} \tau}$. Differentiating $G(L(\epsilon))=\tau \epsilon$ gives $L^{\prime}(0)=\tau U(0)$ and, from $(2.21)$, $P^{\prime}(0)=\tau^{2} D e^{-\mu_{b}^{\text {juv }} \tau}\left(\mu_{b}^{\text {juv }}-\mu_{\text {fly }}^{\text {juv }}(0)\right)$. Therefore, for small $\epsilon$,

$$
P(\epsilon) \approx e^{-\mu_{b}^{\mathrm{juv}} \tau}+\epsilon \tau^{2} D e^{-\mu_{b}^{\mathrm{juv}} \tau}\left\{\mu_{b}^{\mathrm{juv}}-\mu_{\mathrm{fly}}^{\mathrm{juv}}(0)\right\}+\cdots .
$$

From $(2.22)$ we can calculate $Q^{\prime}(0)$ in terms of $p^{*^{\prime}}(0)$, and the latter can be found from $(2.20)$. This gives us the expansion

$$
Q(\epsilon) \approx e^{-\mu_{b}^{\mathrm{juv}} \tau}+\epsilon \tau D e^{-\mu_{b}^{\mathrm{juv}} \tau}\left\{\tau\left(\mu_{b}^{\mathrm{juv}}-\mu_{\mathrm{fly}}^{\mathrm{juv}}(0)\right)-1\right\}+\cdots
$$

Expressions (2.23) and (2.24) are very similar but are not identical. We know the Laplace transform approach to the derivation of the maturation rate introduces approximations. The probabilistic approach is imperfect too in that, mathematically, $n$ in (2.17) might not be an integer. Nonetheless, the closeness of the survival probabilities as given by (2.23) and (2.24) for small $\epsilon$ does at least support the use of expression (2.19) (and, therefore, the model equation (2.15)) for small $\epsilon$, in other words, in situations where the duration of a single migration is small compared to the total maturation time for the bird species.

\section{Model analysis}

We analyse model (2.15) with a view to obtaining conditions for the extinction or survival of the bird species, always assuming that $B(0)=0$ and $B^{\prime}(0)>0$ with further conditions added where necessary. Our first result is on positivity and boundedness of solutions of $(2.15)$. The assumption that $B(\cdot)$ is bounded is realistic. For example, one might think of the case when $B(S)=P S e^{-k S}$, implying that the per-capita egg-laying rate is decreasing. Indeed, individual birds may reduce their clutch sizes when they estimate that the local population density is high, since over-crowding will make it more difficult for the birds to find enough food for their offspring. This point is discussed in Dawkins [2]. 
Proposition 3.1. Suppose that $B(0)=0, B(S)>0$ when $S>0$ and $\sup _{S \geq 0} B(S)<\infty$. Suppose also that $S_{b}^{\mathrm{ad}}(\theta) \geq 0$ on the interval $\theta \in[-\tau-G(L), 0]$ and $S_{b}^{\mathrm{ad}}(0)>0$. Then the solution $S_{b}^{\mathrm{ad}}(t)$ of (2.15) is positive for all $t>0$ and remains bounded.

Proof. Positivity is easily shown by the method of steps (as explained later, in the proof of Theorem 3.4). To establish the boundedness, assume for a contradiction that $S_{b}^{\text {ad }}(t)$ is unbounded. Then there is a sequence $t_{n} \rightarrow \infty$ such that $S_{b}^{\text {ad }}\left(t_{n}\right) \rightarrow \infty$ and, for each $n, \dot{S}_{b}^{\text {ad }}\left(t_{n}\right) \geq 0$ and $S_{b}^{\text {ad }}(t) \leq S_{b}^{\text {ad }}\left(t_{n}\right)$ for all $t \leq t_{n}$. Since we assume $B(\cdot)$ is bounded, those terms in $(2.15)$ that involve $B(\cdot)$ are also bounded and therefore there exists $C>0$ such that

$$
\dot{S}_{b}^{\mathrm{ad}}(t) \leq C-\left(\mu_{b}^{\mathrm{ad}}+D\right) S_{b}^{\mathrm{ad}}(t)+D \exp \left(-\int_{0}^{L} \frac{\mu_{\mathrm{fly}}^{\mathrm{ad}}(\bar{x})}{U(\bar{x})} d \bar{x}\right) S_{b}^{\mathrm{ad}}(t-G(L)) .
$$

Evaluating at $t=t_{n}$, and bounding the exponential by 1 ,

$$
\begin{aligned}
\dot{S}_{b}^{\mathrm{ad}}\left(t_{n}\right) & \leq C-\left(\mu_{b}^{\mathrm{ad}}+D\right) S_{b}^{\mathrm{ad}}\left(t_{n}\right)+D S_{b}^{\mathrm{ad}}\left(t_{n}-G(L)\right) \\
& \leq C-\left(\mu_{b}^{\mathrm{ad}}+D\right) S_{b}^{\mathrm{ad}}\left(t_{n}\right)+D S_{b}^{\mathrm{ad}}\left(t_{n}\right)=C-\mu_{b}^{\mathrm{ad}} S_{b}^{\mathrm{ad}}\left(t_{n}\right) .
\end{aligned}
$$

Therefore, for $n$ sufficiently large, $\dot{S}_{b}^{\text {ad }}\left(t_{n}\right)<0$ which is a contradiction.

Our next result concerns the linear stability of the extinction steady state of (2.15).

Theorem 3.2. Suppose that $B(0)=0, B^{\prime}(0)>0$ and

$$
\begin{aligned}
& \mu_{b}^{\mathrm{ad}}+D>\frac{B^{\prime}(0) e^{p^{*} \tau}}{1+G(L)\left(p^{*}+\mu_{b}^{\mathrm{juv}}+D\right)} \\
& +D \int_{\tau}^{\tau+G(L)} \frac{B^{\prime}(0) e^{p^{*}(a-G(L))}}{1+G(L)\left(p^{*}+\mu_{b}^{\mathrm{juv}}+D\right)} \exp \left(-\int_{0}^{L} \frac{\mu_{\mathrm{fly}}(a-G(L)+G(\bar{x}), \bar{x})}{U(\bar{x})} d \bar{x}\right) d a \\
& +D \exp \left(-\int_{0}^{L} \frac{\mu_{\mathrm{fly}}^{\mathrm{ad}}(\bar{x})}{U(\bar{x})} d \bar{x}\right)
\end{aligned}
$$

with $p^{*}$ given by (2.13). Then the zero solution of (2.15) is locally asymptotically stable, so that small introductions of the birds go extinct.

Proof. The linearisation of (2.15) about its zero solution has the same form as (2.15) itself, but with $B\left(S_{b}^{\text {ad }}(t-\tau)\right)$ replaced by $B^{\prime}(0) S_{b}^{\text {ad }}(t-\tau)$ and $B\left(S_{b}^{\text {ad }}(t-a)\right)$ replaced by $B^{\prime}(0) S_{b}^{\text {ad }}(t-a)$. The delayed terms in the linearised equation have positive coefficients which simplifies things by implying that the linear stability of the zero solution of (2.15) is determined by the real roots of the characteristic equation of the linearisation (Smith [6], Theorem 5.5.1). That characteristic equation, which results from a search for solutions of the linearised equation of the form $S_{b}^{\mathrm{ad}}(t)=\exp (\lambda t)$, is

$$
\begin{aligned}
& \lambda+\mu_{b}^{\text {ad }}+D=\frac{B^{\prime}(0) e^{p^{*} \tau} e^{-\lambda \tau}}{1+G(L)\left(p^{*}+\mu_{b}^{\mathrm{juv}}+D\right)} \\
& +D \int_{\tau}^{\tau+G(L)} \frac{B^{\prime}(0) e^{p^{*}(a-G(L))} e^{-\lambda a}}{1+G(L)\left(p^{*}+\mu_{b}^{\mathrm{juv}}+D\right)} \exp \left(-\int_{0}^{L} \frac{\mu_{\mathrm{fly}}(a-G(L)+G(\bar{x}), \bar{x})}{U(\bar{x})} d \bar{x}\right) d a \\
& +D \exp \left(-\int_{0}^{L} \frac{\mu_{\mathrm{fly}}^{\text {ad }}(\bar{x})}{U(\bar{x})} d \bar{x}\right) e^{-\lambda G(L)} .
\end{aligned}
$$

Complex roots $\lambda$ of (3.2) exist, but the theory in [6] assures us that the dominant root is real. As functions of $\lambda \in \mathbb{R}$, the left hand side of (3.2) is increasing while the right hand side is decreasing. Condition (3.1) 
implies that the left hand side exceeds the right hand side when $\lambda=0$. It follows that all real roots of (3.2) are negative and the theorem is proved.

Next we explore the ecological implications of the extinction condition, inequality (3.1). We summarise our findings in Theorem 3.3. The condition $\tau>G(L)$ is perfectly natural since it just states that the maturation age exceeds the total duration of a single migration from the breeding location back to itself. That duration must be less than one year, and migratory bird species generally take at least one year to mature. Both (i) and (ii) of Theorem 3.3 are concerned with the case when the breeding season is relatively short, due to the assumption of largeness of $D$. Recall that $D$ is the per-capita departure rate of birds from the breeding patch, so that $1 / D$ is the mean time that birds spend in that patch. Statement (i) of Theorem 3.3 is easy to interpret: if birds spend little time in the breeding patch then, all other things being equal, they go extinct because they fail to lay enough eggs. But if time spent in the breeding patch is extremely low then it arguably makes more sense to work with a parameter $\mathcal{P}$ defined as the mean number of eggs laid per bird over the whole breeding season, rather than with $B^{\prime}(0)$ which is the per-capita egg laying rate (at low densities). Since the mean time spent in the breeding patch is $1 / D$, we have $\mathcal{P}=B^{\prime}(0) / D$ so that $B^{\prime}(0)=D \mathcal{P}$ suggesting that $B^{\prime}(0)$ will in practice depend on $D$. Statement (ii) of Theorem 3.3 is, like (i), concerned with the situation when $D$ is large. The difference between (i) and (ii) is that (ii) recognises that, if $D$ is large, the per-capita egg laying rate $B^{\prime}(0)$ would in practice depend on $D$ through the formula $B^{\prime}(0)=D \mathcal{P}$ just derived. This is why, in (ii), the additional extinction condition (3.3) is needed. Note that (3.3) does not involve the breeding patch mortalities $\mu_{b}^{\text {juv }}$ and $\mu_{b}^{\text {ad }}$. This is because if birds spend very little time in the breeding patch then their survival depends mainly on the flyway mortality function $\mu_{\text {fly }}$ defined in (2.8).

Theorem 3.3. Suppose that $B(0)=0, B^{\prime}(0)>0$ and $\tau>G(L)$. Then the extinction steady state solution $S_{b}^{\mathrm{ad}}(t) \equiv 0$ of (2.15) is locally asymptotically stable in each of the following circumstances:

(i) $D$ is sufficiently large and the per-capita egg laying rate $B^{\prime}(0)$ and other parameters are independent of $D$;

(ii) the breeding season is extremely short and

$$
\begin{aligned}
\frac{\mathcal{P}}{G(L)} \int_{\tau}^{\tau+G(L)} & \exp \left(-\frac{(a-G(L))}{G(L)} \int_{0}^{L} \frac{\mu_{\mathrm{fly}}^{\mathrm{juv}}(\bar{x})}{U(\bar{x})} d \bar{x}-\int_{0}^{L} \frac{\mu_{\mathrm{fly}}(a-G(L)+G(\bar{x}), \bar{x})}{U(\bar{x})} d \bar{x}\right) d a \\
& +\exp \left(-\int_{0}^{L} \frac{\mu_{\mathrm{fly}}^{\mathrm{ad}}(\bar{x})}{U(\bar{x})} d \bar{x}\right)<1,
\end{aligned}
$$

where $\mathcal{P}$ is the mean number of eggs laid per bird over the whole breeding season.

Proof. To prove (i), note that (3.1) can be rewritten in the form

$$
\mu_{b}^{\mathrm{ad}}+D\left\{1-\exp \left(-\int_{0}^{L} \frac{\mu_{\mathrm{fly}}^{\mathrm{ad}}(\bar{x})}{U(\bar{x})} d \bar{x}\right)-\varphi(D)\right\}>\frac{B^{\prime}(0) e^{p^{*} \tau}}{1+G(L)\left(p^{*}+\mu_{b}^{\mathrm{juv}}+D\right)},
$$

where

$$
\varphi(D)=\int_{\tau}^{\tau+G(L)} \frac{B^{\prime}(0) e^{p^{*}(a-G(L))}}{1+G(L)\left(p^{*}+\mu_{b}^{\mathrm{juv}}+D\right)} \exp \left(-\int_{0}^{L} \frac{\mu_{\mathrm{fly}}(a-G(L)+G(\bar{x}), \bar{x})}{U(\bar{x})} d \bar{x}\right) d a .
$$

Note that $p^{*}$, which is given by (2.13), depends on $D$. Since $p^{*}<0$ and $p^{*}+\mu_{b}^{\text {juv }}+D>0$ (by $(2.13)$ ), it follows that the right hand side of (3.4) is bounded above by $B^{\prime}(0)$. We now claim that the left hand side of (3.4) grows unboundedly with $D$, and this is clearly the case if $\lim _{D \rightarrow \infty} \varphi(D)=0$. We now prove the latter. To estimate the integrand of the integral that defines $\varphi(D)$, note that $a-G(L)>a-\tau>0$ 
and therefore $e^{p^{*}(a-G(L))}<1$. Also, by $(2.13)$,

$$
p^{*}+\mu_{b}^{\mathrm{juv}}+D=D e^{-p^{*} G(L)} \exp \left(-\int_{0}^{L} \frac{\mu_{\mathrm{fly}}^{\mathrm{juv}}(\bar{x})}{U(\bar{x})} d \bar{x}\right)>D \exp \left(-\int_{0}^{L} \frac{\mu_{\mathrm{fly}}^{\mathrm{juv}}(\bar{x})}{U(\bar{x})} d \bar{x}\right)
$$

since $p^{*}<0$. The integrand is therefore bounded by

$$
\frac{B^{\prime}(0)}{1+G(L) D \exp \left(-\int_{0}^{L} \mu_{\mathrm{fly}}^{\mathrm{juv}}(\bar{x}) / U(\bar{x}) d \bar{x}\right)}
$$

and so $\lim _{D \rightarrow \infty} \varphi(D)=0$. Therefore (3.4) holds for $D$ sufficiently large and (i) is proved.

To prove (ii) we show that, when $B^{\prime}(0)=D \mathcal{P}$, inequality (3.1) reduces to (3.3) when $D$ is very large. We claim that the root $p^{*}$ of (2.13) satisfies

$$
\lim _{D \rightarrow \infty} p^{*}=-\frac{1}{G(L)} \int_{0}^{L} \frac{\mu_{\mathrm{fly}}^{\mathrm{juv}}(\bar{x})}{U(\bar{x})} d \bar{x} .
$$

Indeed, equation (2.13) has the structure $p^{*}+a_{1}+D=D e^{-\left(a_{2} p^{*}+a_{3}\right)}$ or, rearranging so that $D$ appears in one place only, $1+\left(p^{*}+a_{1}\right) / D=e^{-\left(a_{2} p^{*}+a_{3}\right)}$. Sketches of the graphs of the left and right hand sides of this equation against $p^{*}$ for various increasing $D$ reveal that, as $D \rightarrow \infty, p^{*}$ approaches the root of $1=e^{-\left(a_{2} p^{*}+a_{3}\right)}$, leading to $(3.5)$.

With $B^{\prime}(0)=D \mathcal{P}$, dividing inequality (3.1) by $D$, letting $D \rightarrow \infty$ and using (3.5), we arrive at inequality (3.3).

Reversal of the strict inequality in (3.1) will result in instability of the zero solution of (2.15), since in this case the characteristic equation has a real positive root. In this situation, we can also prove that solutions strongly and uniformly persist in the sense described in Theorem 3.4. In that theorem, boundedness of $S_{b}^{\text {ad }}(t)$ is taken as a hypothesis; sufficient conditions for the boundedness are stated in Proposition 3.1.

Theorem 3.4. Suppose that $B(\cdot)$ is twice differentiable, $B(0)=0$ and $B(A)>0$ when $A>0$. Suppose also that $S_{b}^{\mathrm{ad}}(t)$ remains bounded for all $t$ and that

$$
\begin{aligned}
& \mu_{b}^{\mathrm{ad}}+D<\frac{B^{\prime}(0) e^{p^{*} \tau}}{1+G(L)\left(p^{*}+\mu_{b}^{\mathrm{juv}}+D\right)} \\
& +D \int_{\tau}^{\tau+G(L)} \frac{B^{\prime}(0) e^{p^{*}(a-G(L))}}{1+G(L)\left(p^{*}+\mu_{b}^{\mathrm{juv}}+D\right)} \exp \left(-\int_{0}^{L} \frac{\mu_{\mathrm{fly}}(a-G(L)+G(\bar{x}), \bar{x})}{U(\bar{x})} d \bar{x}\right) d a \\
& +D \exp \left(-\int_{0}^{L} \frac{\mu_{\mathrm{fly}}^{\mathrm{ad}}(\bar{x})}{U(\bar{x})} d \bar{x}\right) .
\end{aligned}
$$

Suppose also that $S_{b}^{\mathrm{ad}}(\theta) \geq 0$ on the interval $\theta \in[-\tau-G(L), 0]$ and $S_{b}^{\mathrm{ad}}(0)>0$. Then the solution $S_{b}^{\mathrm{ad}}(t)$ of (2.15) is uniformly strongly persistent, i.e. there exists $\epsilon>0$, independent of the initial conditions, such that $S_{b}^{\text {ad }}(t)$ satisfies

$$
\liminf _{t \rightarrow \infty} S_{b}^{\mathrm{ad}}(t) \geq \epsilon
$$

Proof. To simplify notation we prove the stated persistence property under the condition

$$
\alpha_{1} B^{\prime}(0)+\alpha_{2} B^{\prime}(0) \int_{\tau_{1}}^{\tau_{1}+\tau_{2}} f(a) d a+\alpha_{3}>\mu
$$


for the variable $A(t)$ (to be identified with $S_{b}^{\text {ad }}(t)$ ) satisfying the equation

$$
\frac{d A(t)}{d t}=\alpha_{1} B\left(A\left(t-\tau_{1}\right)\right)+\alpha_{2} \int_{\tau_{1}}^{\tau_{1}+\tau_{2}} f(a) B(A(t-a)) d a+\alpha_{3} A\left(t-\tau_{2}\right)-\mu A(t),
$$

under the assumption that the parameters in (3.8) are positive and $f(a)>0$ for all $a$. For suitably chosen parameters, (3.8) becomes (2.15) and (3.7) becomes (3.6).

Non-negativity of $A(t)$ on its initial interval, and the assumption that $A(0)>0$, enables strict positivity to be easily shown with the aid of the method of steps, beginning with the interval $t \in\left[0, \min \left(\tau_{1}, \tau_{2}\right)\right]$ on which $A^{\prime}(t) \geq-\mu A(t)$ and therefore $A(t) \geq A(0) e^{-\mu t}>0$ for such $t$. By the method of steps, $A(t)>0$ for all later $t$ as well.

Now let $A^{\phi}(t)$ be the solution of (3.8) corresponding to the initial function $\phi$, i.e. the solution such that $A(\theta)=\phi(\theta)$ when $\theta \in\left[-\tau_{1}-\tau_{2}, 0\right]$, and let

$$
m^{\phi}=\liminf _{t \rightarrow \infty} A^{\phi}(t)
$$

From (3.8),

$$
\begin{aligned}
A(\omega)= & e^{-\mu(\omega-\theta)}\left(A(\theta)+\int_{\theta}^{\omega} e^{\mu(s-\theta)}\left\{\alpha_{1} B\left(A\left(s-\tau_{1}\right)\right)\right.\right. \\
& \left.\left.+\alpha_{2} \int_{\tau_{1}}^{\tau_{1}+\tau_{2}} f(a) B(A(s-a)) d a+\alpha_{3} A\left(s-\tau_{2}\right)\right\} d s\right)
\end{aligned}
$$

for $\omega \geq \theta$. Let $q$ be a real number, to be chosen later to advantage, such that $0<q<1$. To prove the theorem, suppose for a contradiction that there exists a sequence of non-negative initial functions $\phi_{n}$ such that $\lim _{n \rightarrow \infty} m^{\phi_{n}}=0$. There exists $T_{n} \rightarrow \infty$ such that

$$
A^{\phi_{n}}(t) \in\left[q m^{\phi_{n}}, \breve{A} / q\right] \text { for all } t \geq T_{n}-\tau_{1}-\tau_{2},
$$

where $\breve{A}$ is any upper bound on $\lim _{\sup _{t \rightarrow \infty}} A(t)$ (i.e., on $S_{b}^{\text {ad }}(t)$ ). Also, there exists $t_{n}>T_{n}+n$ such that $A^{\phi_{n}}\left(t_{n}\right)<m^{\phi_{n}} / q$. From (3.9),

$$
\begin{aligned}
A^{\phi_{n}}\left(t_{n}\right)= & e^{-\mu\left(t_{n}-T_{n}\right)}\left(A^{\phi_{n}}\left(T_{n}\right)+\int_{T_{n}}^{t_{n}} e^{\mu\left(s-T_{n}\right)}\left\{\alpha_{1} B\left(A^{\phi_{n}}\left(s-\tau_{1}\right)\right)\right.\right. \\
& \left.\left.+\alpha_{2} \int_{\tau_{1}}^{\tau_{1}+\tau_{2}} f(a) B\left(A^{\phi_{n}}(s-a)\right) d a+\alpha_{3} A^{\phi_{n}}\left(s-\tau_{2}\right)\right\} d s\right) \\
= & e^{-\mu\left(t_{n}-T_{n}\right)}\left[A^{\phi_{n}}\left(T_{n}\right)+\left\{\alpha_{1} B\left(A^{\phi_{n}}\left(\sigma_{n}-\tau_{1}\right)\right)\right.\right. \\
& \left.\left.+\alpha_{2} \int_{\tau_{1}}^{\tau_{1}+\tau_{2}} f(a) B\left(A^{\phi_{n}}\left(\sigma_{n}-a\right)\right) d a+\alpha_{3} A^{\phi_{n}}\left(\sigma_{n}-\tau_{2}\right)\right\}\left(\frac{e^{\mu\left(t_{n}-T_{n}\right)}-1}{\mu}\right)\right]
\end{aligned}
$$

for some $\sigma_{n} \in\left[T_{n}, t_{n}\right]$, by the integral mean value theorem. Hence

$$
\begin{aligned}
& A^{\phi_{n}}\left(t_{n}\right)=e^{-\mu\left(t_{n}-T_{n}\right)} A^{\phi_{n}}\left(T_{n}\right)+\left(\frac{1-e^{-\mu\left(t_{n}-T_{n}\right)}}{\mu}\right) \\
& \times\left\{\alpha_{1} B\left(A^{\phi_{n}}\left(\sigma_{n}-\tau_{1}\right)\right)+\alpha_{2} \int_{\tau_{1}}^{\tau_{1}+\tau_{2}} f(a) B\left(A^{\phi_{n}}\left(\sigma_{n}-a\right)\right) d a+\alpha_{3} A^{\phi_{n}}\left(\sigma_{n}-\tau_{2}\right)\right\} .
\end{aligned}
$$

Now, as $n \rightarrow \infty, A^{\phi_{n}}\left(t_{n}\right)<m^{\phi_{n}} / q \rightarrow 0$ and $e^{-\mu\left(t_{n}-T_{n}\right)} A^{\phi_{n}}\left(T_{n}\right) \leq e^{-\mu n} \breve{A} / q \rightarrow 0$. Also, the ratio with denominator $\mu$ approaches $1 / \mu$. Therefore, as $n \rightarrow \infty$, the entire curly bracketed term approaches zero 
and so does each individual term of it, since the terms are positive. Moreover, by another application of the integral mean value theorem,

$$
\int_{\tau_{1}}^{\tau_{1}+\tau_{2}} f(a) B\left(A^{\phi_{n}}\left(\sigma_{n}-a\right)\right) d a=B\left(A^{\phi_{n}}\left(\sigma_{n}-\zeta_{n}\right)\right) \int_{\tau_{1}}^{\tau_{1}+\tau_{2}} f(a) d a
$$

for some $\zeta_{n} \in\left[\tau_{1}, \tau_{1}+\tau_{2}\right], n \in \mathbb{N}$. Recall also that the only zero of $B(A)$ is at $A=0$. Taken together, these facts imply that, as $n \rightarrow \infty$,

$$
A^{\phi_{n}}\left(\sigma_{n}-\tau_{1}\right) \rightarrow 0, \quad A^{\phi_{n}}\left(\sigma_{n}-\zeta_{n}\right) \rightarrow 0, \quad A^{\phi_{n}}\left(\sigma_{n}-\tau_{2}\right) \rightarrow 0 .
$$

Since $q<1$, there exists $\xi>0$ such that $B(A)>q B^{\prime}(0) A$ for all $A \in(0, \xi]$. Moreover, there exists $n_{0}$ such that, for all $n>n_{0}$,

$$
A^{\phi_{n}}\left(\sigma_{n}-\tau_{1}\right)<\xi, \quad A^{\phi_{n}}\left(\sigma_{n}-\zeta_{n}\right)<\xi,
$$

so that, for $n$ sufficiently large, using (3.10),

$$
\begin{aligned}
& B\left(A^{\phi_{n}}\left(\sigma_{n}-\tau_{1}\right)\right)>q B^{\prime}(0) A^{\phi_{n}}\left(\sigma_{n}-\tau_{1}\right) \geq q B^{\prime}(0) q m^{\phi_{n}}, \\
& B\left(A^{\phi_{n}}\left(\sigma_{n}-\zeta_{n}\right)\right)>q B^{\prime}(0) A^{\phi_{n}}\left(\sigma_{n}-\zeta_{n}\right) \geq q B^{\prime}(0) q m^{\phi_{n}}, \\
& A^{\phi_{n}}\left(\sigma_{n}-\tau_{2}\right) \geq q m^{\phi_{n}}, \\
& \frac{1-e^{-\mu\left(t_{n}-T_{n}\right)}}{\mu}>\frac{q}{\mu},
\end{aligned}
$$

with the third of these holding for all $n$. It then follows from (3.11) and (3.12) that

$$
\frac{m^{\phi_{n}}}{q}>\frac{q}{\mu}\left\{\alpha_{1} q B^{\prime}(0) q m^{\phi_{n}}+\alpha_{2} q B^{\prime}(0) q m^{\phi_{n}} \int_{\tau_{1}}^{\tau_{1}+\tau_{2}} f(a) d a+\alpha_{3} q m^{\phi_{n}}\right\},
$$

and therefore that

$$
\mu>q^{3}\left(\alpha_{1} q B^{\prime}(0)+\alpha_{2} q B^{\prime}(0) \int_{\tau_{1}}^{\tau_{1}+\tau_{2}} f(a) d a+\alpha_{3}\right) .
$$

The last inequality will contradict (3.7) if the quantity $q \in(0,1)$, which we can choose to advantage, is chosen sufficiently close to 1 . The proof is complete.

\section{Simulations}

We carried out some numerical simulations to illustrate the dynamical behavior of the number of adult birds in the summer breeding patch $b$, according to (2.15), using the mallard duck as a specific example. The mallard is a dabbling duck which is widely distributed across the Northern and Southern Hemispheres. Mallards form pairs in the autumn. The females make nests and lay eggs in early spring. Clutches comprise 1-13 eggs and incubation lasts 23-30 days [3]. The average life span of the mallard in the wild is 5 to 10 years [3]. It is strongly migratory in the northern parts of its breeding range, and winters farther south. It may winter in Mexico but regularly strays into Central America and the Caribbean between September and May. The average distance traveled by satellite-marked mallards during the spring migration was more than 730 miles. The average distance traveled by individual birds during the autumn migration was almost 875 miles. It is reported in [4] that a mallard (a drake) marked with a GPS satellite transmitter flew more than 500 miles during the spring migration, from Minnesota to Saskatchewan, in just four days.

In our simulations, the results of which are shown in Fig. 2, we took $L=1600$ miles which is the total of the distances flown in the autumn and spring migrations and the length of the wintering patch. The 
births function was chosen as the famous Nicholson's blowflies birth rate $B\left(S_{b}^{\text {ad }}\right)=r S_{b}^{\text {ad }} \exp \left(-S_{b}^{\text {ad }} / k\right)$. The flight speed $U(x)$ was taken as

$$
U(x)=U_{0}\left(1-0.99985 \exp \left\{-(x-L / 2)^{2} / 100^{2}\right\}\right),
$$

where $U_{0}$ is a typical flight speed for the birds when they are not pausing at a wintering or stopover location. With this choice for $U(x)$, flight speed of birds is more or less constant except in (roughly) the interval $x \in(600,1000)$ when flight speed drops off sharply, getting close to zero at $x=L / 2=800$ (the wintering location). As explained earlier, in this way we model pausing of birds at the wintering location by letting their flight speed drop off sharply near to that location. If we take $U_{0}=100$, then the total duration of the entire migration is $G(L)=270$ days. In order to simplify the simulation, we assumed the per-capita mortality rates for juvenile and adult birds along the flyway to be independent of the location, and in fact they were taken as constants.

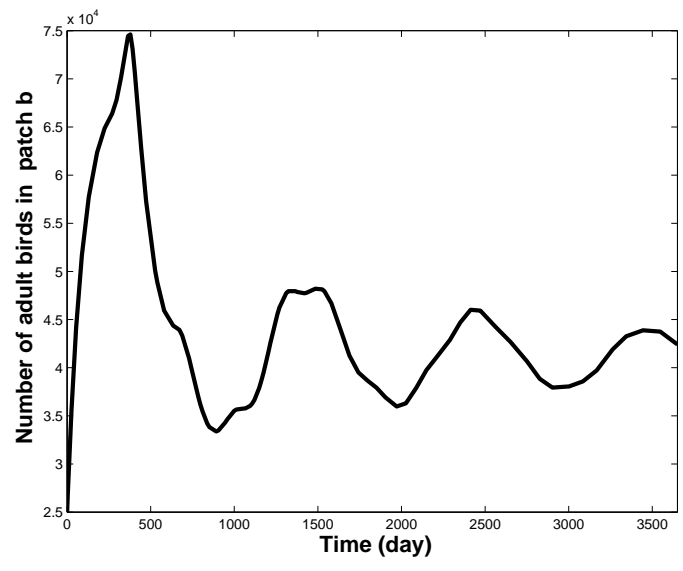

(a) $D=0.01$

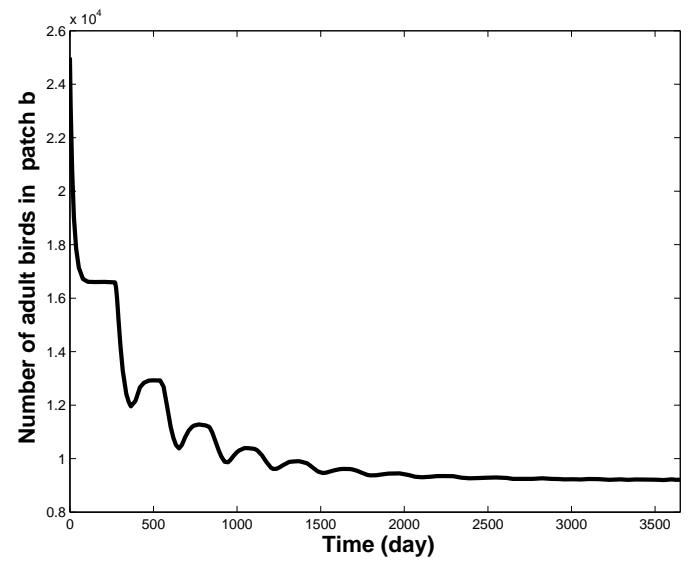

(b) $D=0.05$

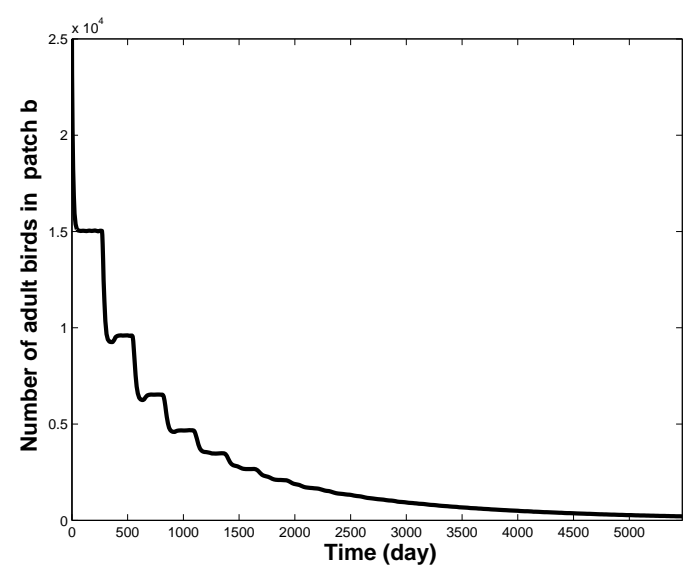

(c) $D=0.1$

Figure 2. Solution $S_{b}^{\text {ad }}(t)$ of (2.15) for various values of $D$, showing a tendency for damped oscillations when $D$ is small, rapid convergence to a non-zero equilibrium for intermediate $D$, and convergence to the zero solution for larger $D$. 


\section{Conclusion}

This paper introduces an approach to modelling the bird migration phenomenon that might be suitable as an alternative, and arguably simpler, approach than we described in some of our previous works (eg. Bourouiba et al [1], Gourley et al [5]). Those previous works used a large number of patches for the breeding, wintering and various stopover sites, and the patches were connected with one dimensional curves representing the migration flyways. Dynamics in patches were described using ordinary differential equations, and dynamics along flyways using reaction-advection equations. The models in [1] and [5] can be reformulated as purely time-dependent systems of delay differential equations, but the order of the system may be large since the number of equations is related to the number of patches.

In the present paper we recognise that locations where birds spend winter, or where birds stop to rest and refuel, can in fact be thought of as particular intervals of the flyway where the flight speed is greatly reduced. Also, in those stopover intervals risks of predation may be greatly increased and per-capita mortality parameters will differ from their values in other parts of the flyway where birds are actually in flight. We have effectively assumed that stopovers, and the wintering location, are just particular flyway intervals through which birds travel very slowly and experience modified risks. These flyway intervals correspond to the two-dimensional regions (patches) in the models of [1] and [5] where birds stop for a while. The approach in the present paper simplifies things by having only one patch (the summer breeding location) and a migration flyway (mathematically, a loop) joining that patch to itself along which all other dynamics take place. The advantage of this approach is that the dynamics can be described by just one delay differential equation for the number of adult birds at the summer breeding location. This equation contains integrals that contain information about conditions encountered at all points along the flyway, such as flight speed and per-capita flyway mortality, including information for intervals of the flyway that are interpreted as stopovers in the sense described above. The ability to reduce the problem to a scalar delay integro-differential equation enables analytical and numerical investigation to take place using well established theories for such equations. The focus of this paper has been on conditions for extinction and for persistence. In particular, we were able to obtain conditions for strong uniform persistence (the strongest kind of persistence result that investigators usually aim for) using an entirely self contained argument. Moreover, the persistence condition is the natural one, reversal of the strict inequality required for the local stability of the extinction state.

Acknowledgements. We thank the referees for their comments.

\section{References}

[1] L. Bourouiba, S.A. Gourley, R. Liu, J. Wu. The Interaction of Migratory Birds and Domestic Poultry and its Role in Sustaining Avian Influenza. SIAM. J. Appl. Maths., 71 (2011), 487-516.

[2] R. Dawkins. The Selfish Gene (30th anniversary edition). Oxford University Press, Oxford, 2006.

[3] N. Drilling, R. Titman, F. McKinney. (2002) Mallard (Anas platyrhynchos). In A. Poole and F. Gill, editors. The Birds of North America. Number 658. The Birds of North America, Philadelphia, Pennsylvania, USA.

[4] Understanding Waterfowl: Tracking the Mallard Migration, http://www.ducks.org/conservation/waterfowlbiology/understanding-waterfowl-tracking-the-mallard-migration/page2.

[5] S.A. Gourley, R. Liu, J. Wu. Spatiotemporal distributions of migratory birds: Patchy models with delay. SIAM. J. Applied Dynamical Systems, 9 (2010), 589-610.

[6] H.L. Smith. Monotone Dynamical Systems. An introduction to the theory of competitive and cooperative systems. Mathematical Surveys and Monographs, Vol 41. American Mathematical Society, Providence, RI, 1995. 